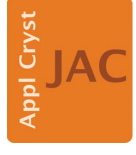

JOURNAL OF APPLIED CRYSTALLOGRAPHY

ISSN 1600-5767

eywords: book reviews; probability

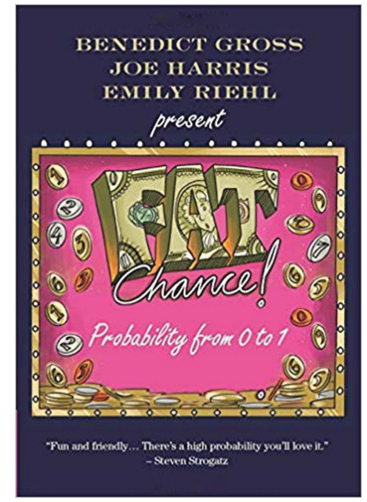

(C) 2019 International Union of Crystallography

\section{Fat Chance! Probability from 0 to 1. By Benedict Gross, Joe Harris and Emily Riehl. Cambridge University Press, 2019. Pp. Xi+200. Paperback price GBP 19.99, ISBN 9781108728188. Hardback price GBP 49.99, ISBN 9781108482967. Ebook price USD 21.00, ISBN 9781108598705.}

\author{
Massimo Nespolo*
}

Université de Lorraine, CNRS, CRM2, Nancy, France. *Correspondence e-mail: massimo.nespolo@univ-lorraine.fr

Fat Chance! is an agile booklet aiming at presenting the theory of probability to a nonspecialized public with minimal calculus background. It is co-authored by three experts, two from Harvard and one from Johns Hopkins, which is an assurance of rigorousness. As explained in the Preface, it takes its origins in a course of the program of General Education at Harvard College, which developed into an online course with a time commitment of 3-5 h per week, available for free (https://online-learning.harvard.edu/ course/fat-chance-probability-ground). The book is intended as a companion to the online course as well as 'for the general reader who wants to learn the basics of probability'.

The level is introductory and the presentation is reader friendly, in a classical American style strikingly contrasting with the austere approach typical of Latin or German tradition. Students and readers without a specific background will not be scared when browsing the pages. A lot of 'gaming' and 'gambling' is used to introduce concepts and illustrate examples: pretty easy to follow although at the end one may get a bit tired of gaming (to be fair, other examples, like school elections, are also used here and there).

Fat Chance! is composed of 14 chapters divided in three parts plus an interlude, followed by two appendices: one containing all the formulas from the text, with a reference to the pages where they showed up (excellent idea), and the other presenting the normal table, i.e. the values of the cumulative distribution function of the normal distribution.

The first part has the simple title Counting and is composed of five chapters introducing really fundamental concepts like exponential notation, tables of independent combinations, factorials, collections and binomial coefficients, as well as a first flavor of probability in terms of games: flipping coins, rolling dice and playing cards. Being really ignorant of poker and bridge I have certainly not enjoyed some of the examples to their full potential: a reader with some gambling experience will no doubt get more hooked. The content of this part corresponds to the last year of senior high school (at least, of the time when I was that age).

The Interlude prepares the reader for the world of probability. Pascal's triangle (the triangular array of binomial coefficients), the binomial theorem and the Catalan numbers (the sequence of natural numbers central to counting recursively defined objects), as well as their geometric interpretation, are the object of the two chapters of the Interlude.

The second part is titled Probability and is composed of four chapters: Expected value, Conditional probability, Life's like that: unfair coins and loaded dice (to address the problem of events that do not have the same occurrence probability) and Geometric probability. As anticipated, games, gambling and elections are repeatedly used to keep the reader bound on Earth rather than floating among the clouds of abstract mathematics (a nice 'zombie apocalypse' example deserves special mention). Particularly appreciated is the emphasis put on drawing the reader's attention to the common trap that results when ignoring Bayes' theorem, which describes the probability of an event based on prior knowledge of conditions that might be related to the event.

The third and last part has the title Probability at large and introduces the law of large numbers and the normal distribution, once again thorough the example of games.

Each chapter includes a series of problems (with solutions) and exercises (without solutions; I could not find online supplementary material). 
The index spans two pages and should be sufficient to trace back every topic dealt with in the book.

There are a few typos spread here and there, which however do not represent an obstacle to the understanding of the content. 13 is easily understood as a typo for 12 at p. 41 . Something seems wrong at p. 117, where we read 'since $k$ is less than $n$, we see that $k n-k$ is less than $k n-n$ ': the correct conclusion is the opposite. The other (few) typos are really minor.
In conclusion, Fat Chance! is an enjoyable small introductory book that, without the pretension of serving as a reference textbook, will certainly help undergraduate students to approach the fascinating world of probability but will also be appreciated by whoever desires to learn the basics through self-education. To obtain the most from it, it is certainly recommended to spend enough time on the problems and exercises, and also to take the online free course as a companion of which this book has been conceived. 